OPEN ACCESS

Edited by:

Murat Akova,

Hacettepe University, Turkey

Reviewed by:

Ahmet Cagkan Inkaya, Hacettepe University, Turkey Siran Keske,

Koç University, Turkey

*Correspondence:

Guangfu Wen

18940258250@163.com

Lin Tao

taolindr@163.com

†These authors have contributed equally to this work

Specialty section:

This article was submitted to Infectious Diseases - Surveillance,

Prevention and Treatment,

a section of the journal

Frontiers in Medicine

Received: 12 August 2021 Accepted: 10 November 2021 Published: 02 December 2021

Citation:

Wang J, Zhu K, Xue Y, Wen G and Tao L (2021) Research Progress in the Treatment of Complications and Sequelae of COVID-19. Front. Med. 8:757605. doi: 10.3389/fmed.2021.757605

\section{Research Progress in the Treatment of Complications and Sequelae of COVID-19}

\author{
Jinpeng Wang ${ }^{1 \dagger}$, Kuoyun Zhu ${ }^{1 \dagger}$, Yuchuan Xue ${ }^{2 t}$, Guangfu Wen ${ }^{3 * t}$ and Lin Tao ${ }^{1 * t}$ \\ ${ }^{1}$ Department of Orthopedics, First Hospital of China Medical University, Shenyang, China, ${ }^{2}$ The First Department of Clinical \\ Medicine, China Medical University, Shenyang, China, ${ }^{3}$ Department of Pediatrics, Shengjing Hospital of China Medical \\ University, Shenyang, China
}

With the improvement in the understanding of COVID-19 and the widespread vaccination of COVID-19 vaccines in various countries, the epidemic will be brought under control soon. However, multiple viruses could result in the post-viral syndrome, which is also common among patients with COVID-19. Therefore, the long-term consequences and the corresponding treatment of COVID-19 should be the focus in the post-epidemic era. In this review, we summarize the therapeutic strategies for the complications and sequelae of eight major systems caused by COVID-19, including respiratory system, cardiovascular system, neurological system, digestive system, urinary system, endocrine system, reproductive system and skeletal complication. In addition, we also sorted out the side effects reported in the vaccine trials. The purpose of this article is to remind people of possible complications and sequelae of COVID-19 and provide robust guidance on the treatment. It is extremely important to conduct long-term observational prognosis research on a larger scale, so as to have a comprehensive understanding of the impact of the SARS-CoV-2 on the human body and reduce complications to the greatest extent.

Keywords: COVID-19, complication, sequelae, eight major systems, treatment

\section{INTRODUCTION}

Since it was first reported in December 2019, the severe acute respiratory syndrome coronavirus 2 (SARS-CoV-2) is still prevalent globally (1). The number of people recovering from COVID-19 continues to rise, and this encouraging result gives people hope. However, are the survivors of COVID-19 really healthy? Could the human body completely return to normal when the viruses are cleared? Recent studies have shown that many patients with mild to moderate COVID-19 may become "long-term victims," and one-third of mild patients will still suffer from lasting sequelae after recovery. It was also observed that $76 \%$ of COVID-19 survivors were troubled with at least one symptom at 6 months after symptom onset, which was even more common among females (2).

It is not clear why COVID-19 has these lasting effects. Many viruses can result in the so-called "post-viral syndrome," which is described as health problems that persist after the viruses are cleared from the body. These are the result of inflammation or other damage that occurs when the immune system resists infection. The respiratory system is the primary target of SARS-CoV2 which is transmitted mainly through the respiratory tract. The main clinical manifestations are related to the symptoms of pneumonia, characterized by severe acute respiratory distress 
syndrome (3). Meanwhile, almost all of the organs are at high risk of being attacked by SARS-CoV-2 $(4,5)$. Various unusual symptoms are observed in patients with COVID-19 which ranges across the body, such as thrombosis, multiple organ failure, immune defects and complications in the immune system. According to clinical statistics, the mortality rate of COVID-19 patients with acute kidney injury was three times higher than those without kidney injury and the mortality rate of patients with cardiovascular system complications is also relatively higher $(6,7)$. In addition, it is also common for COVID-19 patients with complications of the nervous system, digestive system, endocrine system, reproductive system and motor system (8-12).

Therefore, the prevention and treatment of the complications and sequelae of COVID-19 should be the focus in the postepidemic era. In this study, we systematically summarize the treatment of complications and sequelae associated with eight major systems in patients with COVID. Additionally, we also sorted out the complications and sequelae reported in the vaccine trials. We believe that our research will help medical workers around the world respond more effectively to the COVID-19 in the post-epidemic era.

\section{RESPIRATORY SEQUELAE}

The respiratory system is the primary target of the SARSCoV-2 attack. The main clinical symptoms of COVID-19 are related to pneumonia, with the characteristics of severe acute respiratory distress syndrome. In addition, it is noted that many patients who have recovered from COVID-19 still suffered from coughing and shortness of breath, and severe cases would even develop extensive pulmonary fibrosis, which could result in severe dyspnea. Supportive treatment is the basis for the treatment of respiratory symptoms in patients with COVID-19. It is important to let patients rest in bed and to provide them with good nutrition. Meanwhile, a timely infusion is necessary to maintain the stability of the internal environment, avoid electrolyte disturbances and ensure an acid-base balance. Oxygen therapy is used for mild and ordinary cases to relieve difficult breathing, while a ventilator is required to ensure unobstructed breathing for critical patients $(13,14)$. In addition, it was noted by Han X et al. that survivors with severe COVID-19 are at the high risk for post-COVID fibrosis, but there are still no effective therapeutic strategies for pulmonary fibrosis induced by COVID-19 $(15,16)$. Several anti-fibrosis drugs have entered clinical trials, such as Nintedanib (NCT04619680; NCT04541680 and NCT04338802) and Pirfenidone (NCT04282902 and NCT04607928). Meanwhile, IN01 vaccine (NCT04537130), a hybrid of the recombinant epidermal growth factor and cholera toxin B-subunit domain G33D, has also enter phase Ib clinical trials (16). In extremely severe patients, even if the endotracheal tube is intubated with pure oxygen (100\% oxygen concentration), the partial pressure of oxygen in the body is very low, indicating that there is already severe respiratory failure. Hence, extracorporeal Membrane Oxygenation (ECMO) can be applied to carry out oxygenation through an artificial membrane allowing the lung to recover (17). The use of ECMO was recommended by the World Health Organization (WHO) for COVID-19 patients with severe cardiorespiratory failure (18). According to an international cohort study including 1,035 patients with COVID-19 who received ECMO support, use of ECMO was recommended among patients with refractory COVID-19-related respiratory failure (19). BI 764198 is a potent and selective TRPC6 inhibitor that can reduce lung damage in hospitalized patients with new coronary pneumonia and reduce the risk or severity of acute respiratory complication. A phase II clinical trial of BI 764198 was recently launched. Antimicrobial therapy is also needed while maintaining respiratory function. Antiviral therapy mainly uses anti-influenza and anti-AIDS drugs, but the effect is limited and there is no specific drug (20). At the same time, clinicians use antibacterial drugs to prevent potential risks of co-infection, which should be limited to patients with coinfections and those people developing healthcare-associated infections. Critical patients with severe lung fibrosis may require a lung transplantation. Additionally, the serum levels of antimicrobials of patients receiving antimicrobial treatment should be monitored strictly (21). In short, the treatment of respiratory complication and sequelae is a complicated process. The principle is to kill the pathogenic microorganisms and to maintain normal breathing.

\section{CARDIOVASCULAR COMPLICATION}

Although SARS-CoV-2 attacks the respiratory system, it also has negative effects on cardiovascular systems (22). As such, patients with COVID-19 usually exhibited some clinical manifestations caused by cardiovascular diseases, including myocardial injury, myocarditis, acute coronary syndrome (ACS), acute myocardial infarction (AMI), cardiac arrhythmia and heart failure (23). At present, traditional anti-hypertension drugs are administered routinely to patients with a history of cardiovascular diseases during treatment for COVID-19 and they show no signs of aggravating the condition $(24,25)$. However, there are some key points to be considered. Concerns have been raised about the safety of ACE inhibitors (ACEi) and angiotensin receptor blockers (ARB) during the COVID-19 pandemic, which could upregulate the expression of ACE2 in many tissues, including cardiomyocytes. Since SARS-CoV-2 could bind to ACE2 to access human cells, there is a potential increased risk of developing COVID-19 or a more severe illness in patients who are already undergoing background treatment with $\mathrm{ACEi} / \mathrm{ARB}$. It was observed in a large multicenter cohort study that the prevalent treatment with ACEi/ARB among patients with hypertension is not associated with increased risk of COVID-19 diagnosis, hospital admission or subsequent complications (26). It was also observed in a retrospective study involving 362 patients with hypertension that ACEI/ARB had no significant effect on the severity and outcomes of COVID-19 (27). However, it was also noted that temporary discontinuation of ACEi or ARB had no appreciable effect on the maximum severity of disease within 30 days which, on the contrary, might be associated with a faster and better recovery (28). Therefore, appropriate strategies for the use of ACEi/ARB among COVID-19 patients with hypertension 
should be evaluated on individual basis such as the progression of diseases, indications for ACEi/ARB and the clinical feasibility of replacement therapies (28). In addition, various antiretroviral medications have significant interactions with cardiac drugs, which should be considered and an appropriate dose change also needs to be changed (29). ECMO is also recommend for patients with cardiac failure secondary to COVID-19 (30). However, coagulation abnormalities is common among critically ill patients with COVID-19, including thrombotic microangiopathy and venous and arterial thromboembolic complications (31). Meanwhile, ECMO usually leads to hypercoagulation status of patients. Hence, it is crucial to evaluate the coagulation status of COVID-19 patients who received ECMO and also dosages of anticoagulation drugs (32).

\section{NEUROLOGICAL COMPLICATION}

Neurological symptoms, including meningitis, encephalitis, myelitis, acute disseminated encephalomyelitis and stroke, are also common in patients with COVID-19 and these patients might suffer from severe neurological sequelae (33). It is recommended for patients with cerebrovascular-related symptoms to use antihypertensive drugs, angiotensin-converting enzyme inhibitors (ACEI) and angiotensin II receptor blockers (ARB) in order to increase the expression of ACE2 (34). However, patients with hypertension may need to switch to calcium channel blockers (CCB) and diuretics (35). In addition, the patients with intracranial infections should be treated in terms of the principles of diagnosis and treatment of intracranial infection, protecting against dehydration, controlling seizures and providing antipsychotic treatment (36). In addition, sufficient attention and symptomatic treatment should be given for complication secondary to nerve damage, such as abnormalities of the skeletal muscle, glands and internal organs.

\section{DIGESTIVE COMPLICATION}

The digestive system also presents a high risk of being invaded by SARS-CoV-2 (37). A wide range of digestive symptoms are observed in patients with COVID, including gastrointestinal reactions such as loss of appetite, vomiting, diarrhea and indigestion, as well as liver dysfunction $(9,38)$. Despite the presence of all of the digestive disorders associated with the coronavirus, to date there are no guidelines for the treatment of gastrointestinal symptoms associated with COVID-19. The intestinal flora produces various vitamins, fatty acids, bile acids and immune factors through the fermentation and decomposition of food, and it participates in the regulation of immune function (39). Significant alterations were observed in COVID-19 patients with characteristics of increased opportunistic pathogens and decreased beneficial commensals during hospitalization (40). In addition, it was also noted by $\mathrm{Y}$. Wu et al. that gut microbiota was associated with SARS-Cov-2 virus load, and the microbiome dysbiosis caused by COVID-19 persists still exists even after the viral clearance (41). Therefore, strategies to restore the composition of intestinal flora might reduce the severity of COVID-19 and related complications. Indeed, the prevention and treatment strategies for COVID-19 considering gastroenterology and intestinal microbiota have been of extensive interest. It was proposed in multiple studies that treatment with probiotics might alleviate the progression of COVID-19 (42-44). However, it was demonstrated by Mak JWY et al. that there is no direct evidence for the use of probiotics in COVID-19, and conventional probiotic treatment is not appropriate among patients with COVID-19 (45). Hence, further studies should be focused on the pathogenesis of SARS-CoV-2 and its effect on intestinal microbiota. Meanwhile, whether probiotic treatment could be used as complementary resource for the prevention and restoration of alterations of intestinal microbiota in the absence of direct evidence still warrants further discussion. In addition, ACE2 inhibitors have been found to affect the composition of the intestinal flora and relieve gastrointestinal symptoms in mice by activating mTOR (46). Enteral nutrition can also help restore intestinal digestion, absorption and physiological peristalsis, and maintain the normal function of the gastrointestinal microecology and mucosal immunity. For patients with severe gastrointestinal symptoms of COVID-19, a nutritional risk assessment could be performed (47). Enteral nutrition is the preferred pathway for promoting intestinal integrity and immune function in high-risk populations, including critically ill patients (48). For patients with severe liver damage from COVID-19, drugs that protect the liver, reduce inflammation and reduce jaundice should be used, such as polyene phosphatidyl choline, glycyrrhizic acid, bicyclol, and vitamin E (49). One or two drugs should be selected according to the degree of liver function damage, to avoid aggravating the liver burden and drug interactions.

\section{URINARY COMPLICATION}

After lung infection, SARS-CoV-2 enters the systemic circulation and reaches the kidneys, where it is concentrated and damages resident cells (50). It is observed that kidney involvement in patients with COVID-19 is frequent, which ranges from mild proteinuria to acute kidney injury (AKI) $(51,52)$. In addition, the mortality of patients with AKI is apparently higher than those without kidney damages (6). Therefore, it is necessary to consider all available therapies apart from regular antiviral medications to support renal function. Through daily monitoring and analysis of the patients, clinicians can take potential early intervention measures, including CRRT, as soon as possible to protect renal function, especially for patients with monotonously elevated plasma creatinine (Cre) levels, which may be the key to decrease mortality. Continuous renal replacement therapy (CRRT), a process that mimics the process of glomerular filtration, injects arteriovenous blood into a semipermeable member filter to non-selectively remove the overexpressed inflammatory cytokines from the circulation and block the inflammatory cascade reactions, which subsequently prevents the progression of the disease and improves the prognosis of patients with COVID-19. In addition, systemic anticoagulation 
therapy is also essential for patients receiving CRRT due to the hypercoagulable state of the patients and the high frequency of clotting in CRRT filters (52). The use of convalescent plasma of patients recovered from COVID-19 was traditionally considered to be capable of accelerating clinical recovery and thereby reduce the occurrence of kidney injury. However, it was noted in a multicenter, randomized clinical trial that compared with patients with standard treatment, no significant benefit was observed in clinical improvement at 28 days or mortality among patients who received standard treatment combined with convalescent plasma transfusion therapy (53). It was also observed in a randomized trial that convalescent plasma therapy in addition to standard treatment was not associated with decreased mortality rate or improvement of other clinical outcomes among patients with severe COVID-19. Recently, it was also implicated in a meta-analysis published in JAMA that convalescent plasma administration was not significantly associated with clinical improvement in all-cause mortality or other clinical outcomes (54). Hence, more clinical trials are acquired to establish the optimal conditions for the use of convalescent plasma. Additionally, kidney transplantation is the final choice if the patient's condition requires and permits it.

\section{ENDOCRINE COMPLICATION}

It is indicated that endocrine organs such as pancreas, thyroid, adrenal glands and pituitary are also vulnerable to SARSCoV-2 (55). In addition, COVID-19 could aggravate insulin resistance. Patients with diabetes mellitus are more susceptible to SARS-CoV-2 and are associated with increased mortality (56). For patients with severe comorbidities, terminal illness, or inability to conduct frequent glucose monitoring or close nursing supervision, less aggressive insulin therapy aimed at reducing glucosuria, dehydration, and electrolyte disturbances may be more appropriate (57). Thyrotoxicosis may worsen cardiovascular disease and, in some cases, lead to arrhythmias. Therefore, rapid evaluation of free thyroid hormones and TSH contributes to early diagnosis and appropriate treatment and helps to avoid more serious complication. Moreover, The British Thyroid disorder Association and The Society for Endocrinology have issued a consensus statement on the specific problem of thyroid dysfunction during the COVID-19 pandemic, stating that patients with underlying hypothyroidism or hyperthyroidism are advised to continue taking their prescription drugs as usual (58). In addition, rapid screening of the function of the pituitary-adrenal axis and identification of this condition may lead to appropriate alternative therapy to prevent severe shock. In the presence of subacute thyroiditis or adrenal insufficiency, corticosteroid therapy should be used to interrupt the release of large amounts of thyroid hormone and improve adrenal function, thereby preventing the clinical deterioration of these patients (59).

\section{REPRODUCTIVE COMPLICATION}

There are also obvious gender differences in reproductive complication and sequelae of COVID-19. Symptoms including oligospermia, orchitis, scrotal discomfort and erectile dysfunction are observed in male patients (60-62), while premature birth, fetal distress and premature rupture of fetal membranes are observed in pregnant patients and fetuses with COVID-19 $(63,64)$. In addition, male patients infected with SARS-CoV-2 also exhibited impaired spermatogenesis. It was observed in an observational study that $39.1 \%$ of male patients with COVID-19 have oligospermia and $60.9 \%$ of them have a high inflammatory state in their semen (61). Persistent fever, which is common among COVID-19 patients, would result in temperature changes in the testes and subsequently lead to damage and degeneration of germ cells (65). There is no specific treatment for reproductive dysfunction in patients with COVID-19, but it is important to lower the body temperature to prevent impairment of spermatogenesis due to the fever. Phosphodiesterase-5 inhibitors have been traditionally approved for the treatment of erectile dysfunction since 1998. Surprisingly, sildenafl, the first PDE-5 inhibitor applied in the clinical, has been suggested as a treatment for COVID patients by improving pulmonary function and lowering the risk of vascular injury and thrombotic complication in COVID-19 patients (66). Hence, it is significant to evaluate the effect of other medical interventions on COVID-19 that have a protective function of the reproductive system to reduce the risk of reproductive complication after recovery.

\section{SKELETAL COMPLICATION}

Due to the respiratory system damage caused by SARS-CoV2 , there is an increase in blood lactic acid levels and decrease in blood cell oxygen-carrying capacity. Hence, skeletal muscle hypoxia and ischemia may occur in patients with COVID-19, resulting in myalgia and joint pain (67). In this case, restoring the body's oxygen level will help to relieve the pain. Therefore, when the body's viral load decreases and the oxygen transport function is restored, the pain will be relieved naturally. Active antiviral therapy to restore the respiratory system is the key to alleviating the pain $(68,69)$. Under normal circumstances, patients with skeletal muscle joint pain can use non-steroidal anti-inflammatory drugs (NSAIDs) to relieve pain, and there is no evidence that NSAIDs aggravate COVID-19 (70). So, we may be able to relieve pain symptoms by administering NSAIDs. In addition to the important roles of vitamin $\mathrm{D}$ in bone metabolism, it is also involved in the progression of COVID-19. It was observed by Sulli A et al. that $25 \mathrm{OH}$-vitamin D serum deficiency is associated with more severe pulmonary involvement, increased disease duration and mortality risk among elderly COVID-19 patients (71). It was also noted in a retrospective case-control study that compared with population-based controls, serums levels of 25OH-vitamin D are lower in hospitalized COVID-19 patients, who were at increased risk of Vitamin D deficiency (72). However, Vitamin D supplement among patients with COVID19 is highly controversial. It was observed in a multi-center, randomized clinical trial that A 5000 IU daily oral vitamin D3 supplementation for 2 weeks decreased recovery times for cough and gustatory sensory loss among mild to moderate COVID19 patients with poor vitamin D status (73). Meanwhile, it was also noted by Murai $\mathrm{IH}$ et al. in a randomized controlled trial 


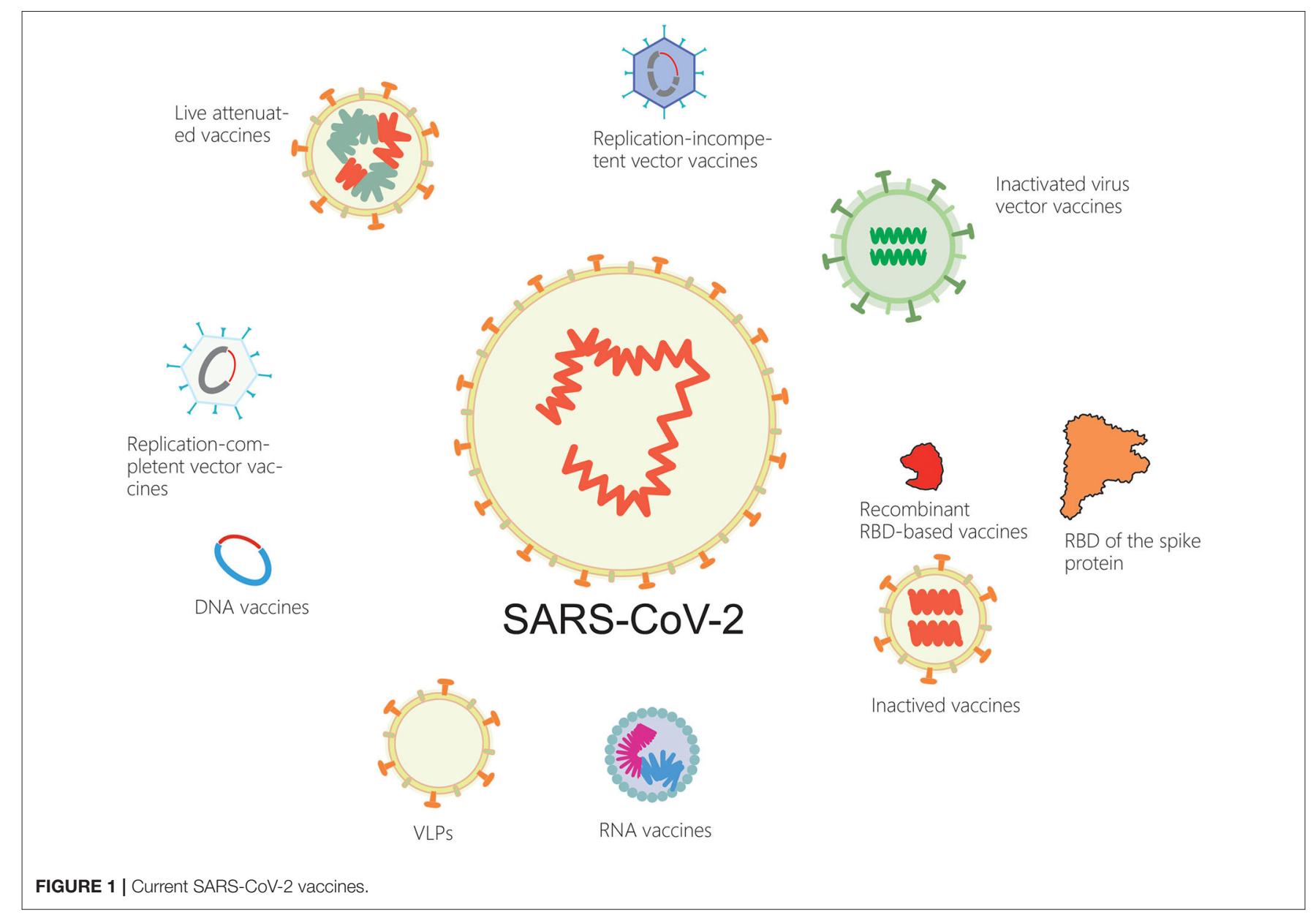

that a single high dose of vitamin D3 did not significantly reduce hospitalization time for COVID-19 patients (74). Hence, so far, vitamin D supplement is recommended for everyone with increased time spent indoors due to strict lockdowns during COVID-19, only in order to maintain bone and muscle health, but there is not sufficient evidences to support vitamin D supplement with the aim of preventing or treating COVID-19 (75). Additionally, staying in bed in the ICU for a long time will cause an imbalance in protein synthesis and degradation, especially in the lower limbs, where muscle protein turnover is reduced, protein synthesis is slow, degradation increases, muscle cells atrophy, and strength decreases. Therefore, patients should minimize the time of intubation to reduce the time of bed rest and engage in appropriate activities to enhance the strength of the skeletal muscles. In SRAS-COV-1 from 2002 to 2004, studies have proved that rehabilitation exercise is beneficial to restore the function of the musculoskeletal system. Survivors of COVID-19, which has a similar pathogenesis, should be able to recover the function of their musculoskeletal system in the same way with the same rehabilitation exercises (76). In China, rehabilitation exercises have been added to treatment guidelines for COVID-19 patients to improve lung function via leisurely activities (77). In addition, pulmonary rehabilitation is also necessary for COVID-19 patients. It was demonstrated that different pulmonary rehabilitation interventions, including combined exercise (aerobic with strength), combined exercise with specific respiratory exercises, and aerobic exercises with specific respiratory muscle training, could obviously improve pulmonary functional and quality of life of patients recovered from severe COVID-19 (78). It was also recommended that rehabilitation care is crucial for patients with severe COVID-19, which should be implemented as soon as possible, and performed at the same time with regular treatment of COVID-19 according to the correct assessment (79).

\section{EFFECTS OF ANTI-INFLAMMATORY DRUGS ON LONG-TERM SEQUELAE OF COVID-19}

Dysregulated inflammatory response during SARS-CoV-2 infection was regarded to be major cause of severity and death of COVID-19 (80). Therefore, anti-inflammatory drugs are widely used during the treatment of COVID-19. In addition to its short-term benefits, use of anti-inflammatory drugs also affect long-term outcomes of COVID-19 patients. According 


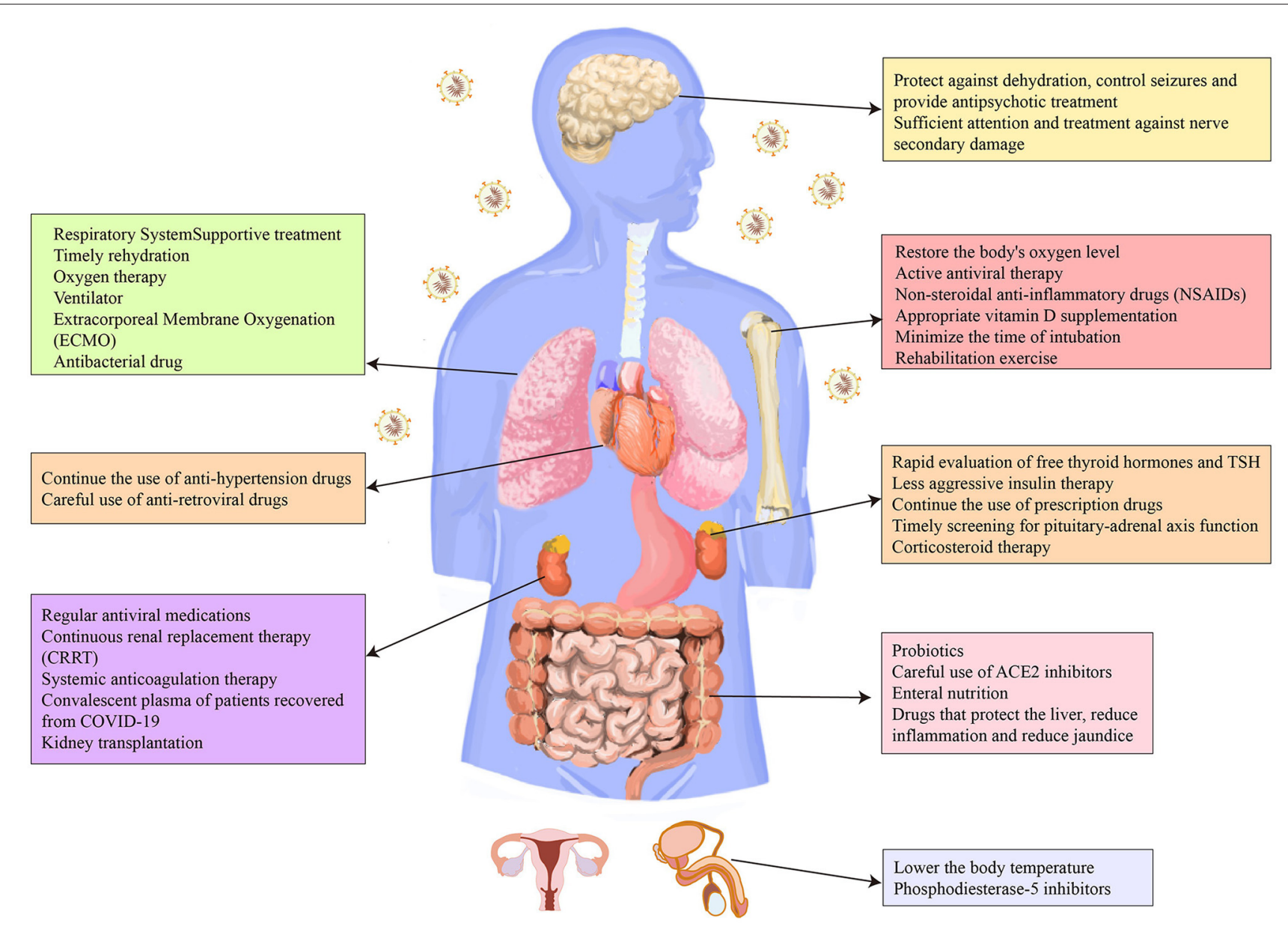

FIGURE 2 | Therapeutic strategies for the complications and sequelae of eight major systems caused by COVID-19.

to current studies, use of anti-inflammatory drugs, such as tocilizumab and NSAIDs, have no adverse effects on the short or long outcomes of COVID-19 (81, 82). In addition, it was demonstrated by Catalán IP et al. that treatment with systemic corticosteroids during hospital admissions is associated with decreased long-term symptoms and improved quality of life, which could be explained by relieving intense inflammation during the acute phase of COVID-19 and subsequently reduce organ and tissue damages caused by SARS-CoV-2 infection. (83). Due to extensive use of anti-inflammatory drugs in the treatment of COVID-19, risk of late-onset complications has always been concerned. Therefore, more long-term follow-up studies of COVID-19 patients treated with various anti-inflammatory drugs are necessary for safety assess and appropriate use of anti-inflammatory drugs among COVID-19 patients.

\section{SIDE EFFECTS OF THE COVID-19 VACCINES}

In the next few months, billions of people around the world will be vaccinated against the new coronavirus. Serval SARSCoV-2 vaccine candidates are currently available, including inactivated virus vaccines, live attenuated vaccines, recombinant protein vaccines, DNA vaccines and RNA vaccines. Other types of vaccines include replication-incompetent vector vaccines, replication-competent vector vaccines, inactivated virus vector vaccines that display the spike protein on their surface (84) (Figure 1).

Ad5-nCoV, a viral vector vaccine which was developed by CanSino Biologics and also the People's Liberation Army, was the first candidate to participate in human trials and is now in clinical phase $2(85,86)$. Having proved to be safe in single blind, randomized phase $1 / 2$ clinical trials, ChAdOx1 which was developed by scientists at Oxford University has already started phase 3 clinical trials (87). In Russia, a heterologous recombinant adenovirus ( $\mathrm{rAd}$ )-based vaccine, Gam-COVIDVac, manufactured by Gamaleya NRCEM (Moscow, Russia) started phase 3 trials and showed $91.6 \%$ efficacy against COVID-19 (88). In addition, a US-based biotechnology firm has developed a DNA-based vaccine named INO-4800, which is uses a relatively more recent vaccine technique. PiCoVacc, which is an inactivated vaccine developed by a Chinese private biopharma company, have currently entered phase 3 trial (89). $\mathrm{BBIBP}-\mathrm{CorV}$ is also an inactivated vaccine in phase $1 / 2$ clinical 
trials which is developed by Beijing Institute of Biological Products (Beijing, China) (90). The Beijing Institute of Biological Products and the Wuhan Institute of Biological Products are also developing some inactivated vaccines, which are in the $2 / 3$ clinical phases. Recently, Clover Biopharmaceuticals (Chengdu, China) developed a s-trimer (S-Trimer) SCB-2019 with two different adjuvants, which triggered obvious humoral and cellular response to SARS-CoV-2 in clinical phase 1 (91). Moreover, two RNA vaccines named mRNA-1273 and BNT-162 are also under development, which could transmit information molecules to guide human body cells and produce the spike proteins of SARS-CoV-2 (92).

The World Health Organization (WHO) maintains a working document that includes most of the vaccines in development and it is available at https://www.who.int/publications/m/item/draftlandscape-of-covid-19-candidate-vaccines. The development of a mRNA vaccine to prevent SARS-CoV-2 infection was successful, and no serious problems occurred during the phase 3 clinical trials that are still ongoing (93). However, clinical trials of the vaccine have shown that the vaccine is not absolutely safe, and some recipients experience different side effects (94). The main symptoms include fever, arm soreness and fatigue. Most scientists think these symptoms are uncomfortable but not dangerous. Pfizer's COVID-19 vaccine shows 95\% safety, but due to the large population receiving the vaccine, a $5 \%$ risk will cause hundreds of thousands of people to have different side effects. There are also occasional severe allergic reactions within a few hours after receiving Pfizer's COVID-19 vaccine. In addition, a vaccine from Australia was also urgently stopped due to false positives for HIV after vaccination. At present, vaccines designed in Europe and China have more advantages in safety, but lack extensive clinical trial data. Each vaccine has more or less unknown side effects and complication, and their long-term sequelae require clinical trials and the test of time. These severe allergic reaction cases raise more questions than the answers given and such safety signals are almost inevitable after a vaccination programme involving millions of people is launched. We need to develop a firm and proactive "safe road map" to clarify the pathogenesis, identify people at risk of such reactions, and adopt strategies that help with treatment and prevention.

\section{DISCUSSION}

The current study indicated that the complication and sequelae of COVID-19 are primarily caused by the patient's immune disorder. In addition, the clinical symptoms and prognosis of SARS-CoV-2 infection are also closely related to immune disorders. Most countries regard elevated IL-6 as a serious and critical clinical warning indicator. According to the clinical data of patients with COVID-19, pro-inflammatory cytokines and chemokines can avoid the risk of a cytokine storm and achieve the possibility of self-healing, which is also the reason why most mild and moderate patients can be cured (95). However, some mild patients also experienced a cytokine storm due to immune disorders. This involves a suicide attack that can damage the virus but also leave behind collateral damage, which will eventually lead to further lung injury, multiple organ failure and even increase the risk of death (96). Therefore, monitoring the level of pro-inflammatory cytokines and taking corresponding intervention measures as early as possible is of great significance to delay the transformation of mild or moderate patients to severe patients and also to achieve good clinical outcomes.

In addition to the therapeutic approaches mentioned above, inflammatory factor blockers (glycyrrhetinic acid, tocilizumab, interferon $-\gamma$ blockers) and stem cell therapy are also expected to become effective means to control cytokines. Additionally, some Chinese medicine prescriptions can also block viruses from entering cells and regulate the body's immune response. Regarding the highly controversial method of reinfusing the serum of recovered COVID-19 patients, although most studies have confirmed that it can enhance immunity, it is difficult to obtain plasma during the recovery period, and its effectiveness may be related to the timing of its extraction. The neutralizing antibody titer in the serum is variable, so its clinical application is also subject to certain restrictions.

At present, the therapy of complications and sequelae of COVID-19 is limited to symptomatic treatment, and there is even no effective treatment for complications or sequelae in certain systems, such as the reproductive system. Therefore, more attention should be focused on the effectiveness and safety of the novel treatment measures of COVID-19, especially the treatment of the complications and sequelae. In addition, with the widespread vaccination of COVID-19 vaccines in various countries, medical workers should always pay attention to the side effects of the vaccine and give timely treatment. We systematically analyzed current treatment for the complications and sequelae of COVID-19 (Figure 2). We also sorted out the side effects reported in the vaccine trials. We believe that our research will provide a theoretical rationale for the management of COVID-19 survivors and thereby improve their prognosis.

\section{AUTHOR CONTRIBUTIONS}

Material preparation, data collection, and analysis were performed by JW, KZ, YX, GW, and LT. The first draft of the manuscript was written by JW, KZ, YX, and LT. All authors commented on previous versions of the manuscript, read and approved the final manuscript, and contributed to the study conception and design.

\section{ACKNOWLEDGMENTS}

Thanks to all the studies that provided data for us. 


\section{REFERENCES}

1. Zhu N, Zhang D, Wang W, Li X, Yang B, Song J, et al. A novel coronavirus from patients with pneumonia in China, 2019. N Engl J Med. (2020) 382:72733. doi: 10.1056/NEJMoa2001017

2. Huang C, Huang L, Wang Y, Li X, Ren L, Gu X, et al. 6-month consequences of COVID-19 in patients discharged from hospital: a cohort study. Lancet. (2021) 397:220-32. doi: 10.1016/S0140-6736(20)32656-8

3. Quan C, Li C, Ma H, Li Y, Zhang H. Immunopathogenesis of coronavirusinduced acute respiratory distress syndrome (ARDS): potential infectionassociated hemophagocytic lymphohistiocytosis. Clin Microbiol Rev. (2020) 34:e00074-20. doi: 10.1128/CMR.00074-20

4. Jamwal S, Gautam A, Elsworth J, Kumar M, Chawla R, Kumar P. An updated insight into the molecular pathogenesis, secondary complications and potential therapeutics of COVID-19 pandemic. Life Sci. (2020) 257:118105. doi: 10.1016/j.lfs.2020.118105

5. Singh Y, Gupta G, Kazmi I, Al-Abbasi FA, Negi P, Chellappan DK, et al. SARS CoV-2 aggravates cellular metabolism mediated complications in COVID-19 infection. Dermatol Ther. (2020) 33:e13871. doi: 10.1111/dth.13871

6. Kolhe NV, Fluck RJ, Selby NM, Taal MW. Acute kidney injury associated with COVID-19: a retrospective cohort study. PLoS Med. (2020) 17:e1003406. doi: 10.1371/journal.pmed.1003406

7. Varshney AS, Wang DE, Bhatt AS, Blood A, Sharkawi MA, Siddiqi HK, et al. Characteristics of clinical trials evaluating cardiovascular therapies for Coronavirus Disease 2019 Registered on ClinicalTrials.gov: a cross sectional analysis. Am Heart J. (2021) 232:105-15. doi: 10.1016/j.ahj.2020.10.065

8. Koralnik IJ, Tyler KL. COVID-19: a global threat to the nervous system. Ann Neurol. (2020) 88:1-11. doi: 10.1002/ana.25807

9. Zhong P, Xu J, Yang D, Shen Y, Wang L, Feng Y, et al. COVID-19-associated gastrointestinal and liver injury: clinical features and potential mechanisms. Signal Transd Target Ther. (2020) 5:256. doi: 10.1038/s41392-02000373-7

10. Freire Santana M, Borba MGS, Baía-da-Silva DC, Val F, Alexandre MAA, Brito-Sousa JD, et al. Case report: adrenal pathology findings in severe COVID-19: an Autopsy Study. Am J Trop Med Hyg. (2020) 103:16047. doi: 10.4269/ajtmh.20-0787

11. Ren X, Wang S, Chen X, Wei X, Li G, Ren S, et al. Multiple expression assessments of ACE2 and TMPRSS2 SARS-CoV-2 entry molecules in the urinary tract and their associations with clinical manifestations of COVID-19. Infect Drug Resist. (2020) 13:3977-90. doi: 10.2147/IDR.S270543

12. Gonzalez A, Orozco-Aguilar J, Achiardi O, Simon F, Cabello-Verrugio C. SARS-CoV-2/renin-angiotensin system: deciphering the clues for a couple with potentially harmful effects on skeletal muscle. Int J Mol Sci. (2020) 21:7904. doi: 10.3390/ijms21217904

13. Lee SG, Park GU. Clinical characteristics and risk factors for fatality and severity in patients with coronavirus disease in korea: a nationwide population-based retrospective study using the Korean Health Insurance Review and Assessment Service (HIRA) database. Int J Environ Res Public Health. (2020) 17:8559. doi: 10.3390/ijerph17228559

14. Khan E, Lal S, Hashmi J, Thomas J, Malik MA. Per-cutaneous dilatation tracheostomy (PCTD) in COVID-19 patients and peri-tracheostomy care: a case series and guidelines. Pak J Med Sci. (2020) 36:17148. doi: 10.12669/pjms.36.7.3518

15. Han X, Fan Y, Alwalid O, Li N, Jia X, Yuan M, et al. Six-month followup chest CT findings after severe COVID-19 pneumonia. Radiology. (2021) 299:E177-86. doi: 10.1148/radiol.2021203153

16. Bazdyrev E, Rusina P, Panova M, Novikov F, Grishagin I, Nebolsin V. Lung Fibrosis after COVID-19: treatment prospects. Pharmaceuticals. (2021) 14:807. doi: 10.3390/ph14080807

17. Zayat R, Kalverkamp S, Grottke O, Durak K, Dreher M, Autschbach R, et al. Role of extracorporeal membrane oxygenation in critically ill COVID19 patients and predictors of mortality. Artif Organs. (2020) 45:E158E170. doi: 10.1055/s-0041-1725788

18. World Health Organization. Clinical Management of Severe Acute Respiratory Infection (SARI) When COVID-19 Disease Is Suspected: Interim Guidance, 13 March 2020. Geneva: World Health Organization (2020).

19. Barbaro RP, MacLaren G, Boonstra PS, Iwashyna TJ, Slutsky AS, Fan E, et al. Extracorporeal membrane oxygenation support in COVID-19: an international cohort study of the Extracorporeal Life Support Organization registry. Lancet. (2020) 396:1071-8. doi: 10.1016/S0140-6736(20)32008-0

20. Rameshrad M, Ghafoori M, Mohammadpour AH, Nayeri MJD, Hosseinzadeh $\mathrm{H}$. A comprehensive review on drug repositioning against coronavirus disease 2019 (COVID-19). Naunyn Schmiedebergs Arch Pharmacol. (2020) 393:113752. doi: 10.1007/s00210-020-01901-6

21. Bakir Ekinci P, Kara E, Er AG, Inkaya AC, Demirkan K, Uzun O. Challenge in treating COVID-19 associate pulmonary aspergillosis: supratherapeutic voriconazole levels. Br J Clin Pharmacol. (2021). doi: 10.1111/bcp.14953. [Epub ahead of print].

22. Inciardi RM, Lupi L, Zaccone G, Italia L, Raffo M, Tomasoni D, et al. Cardiac involvement in a patient with coronavirus disease 2019 (COVID-19). JAMA Cardiol. (2020) 5:819-24. doi: 10.1001/jamacardio.2020.1096

23. Driggin E, Madhavan M, Bikdeli B, Chuich T, Laracy J, Biondi-Zoccai G, et al. Cardiovascular considerations for patients, health care workers, and health systems during the COVID-19 pandemic. J Am Coll Cardiol. (2020) 75:2352-71. doi: 10.1016/j.jacc.2020.03.031

24. Reynolds HR, Adhikari S, Pulgarin C, Troxel AB, Iturrate E, Johnson SB, et al. Renin-angiotensin-aldosterone system inhibitors and risk of COVID-19. N Engl J Med. (2020) 382:2441-8. doi: 10.1056/NEJMoa2008975

25. Patel AB, Verma A. COVID-19 and angiotensin-converting enzyme inhibitors and angiotensin receptor blockers: what is the evidence? JAMA. (2020) 323:1769-70. doi: 10.1001/jama.2020.4812

26. Morales DR, Conover MM, You SC, Pratt N, Kostka K, Duarte-Salles T, et al. Renin-angiotensin system blockers and susceptibility to COVID-19: an international, open science, cohort analysis. Lancet Digit Health. (2021) 3:e98-114. doi: 10.1016/S2589-7500(20)30289-2

27. Li J, Wang X, Chen J, Zhang H, Deng A. Association of renin-angiotensin system inhibitors with severity or risk of death in patients with hypertension hospitalized for coronavirus disease 2019 (COVID-19) infection in Wuhan, China. JAMA Cardiol. (2020) 5:825-30. doi: 10.1001/jamacardio.2020.1624

28. Bauer A, Schreinlechner M, Sappler N, Dolejsi T, Tilg H, Aulinger BA, et al. Discontinuation versus continuation of renin-angiotensin-system inhibitors in COVID-19 (ACEI-COVID): a prospective, parallel group, randomised, controlled, open-label trial. Lancet Respir Med. (2021) 9:86372. doi: 10.1016/S2213-2600(21)00214-9

29. Bansal M, Cardiovascular disease and COVID-19. Diabetes Metab Syndrome Clin Res Rev. (2020) 14:247-50. doi: 10.1016/j.dsx.2020.03.013

30. Lorusso R, Combes A, Coco VL, De Piero ME, Belohlavek J. ECMO for COVID-19 patients in Europe and Israel. Intens Care Med. (2021) 47:3448. doi: $10.1007 / \mathrm{s} 00134-020-06272-3$

31. Levi M, Thachil J, Iba T, Levy JH. Coagulation abnormalities and thrombosis in patients with COVID-19. Lancet Haematol. (2020) 7:e43840. doi: 10.1016/S2352-3026(20)30145-9

32. Zhang Y, Ji B, Zhou Z. ECMO support for COVID-19: a balancing act. Lancet. (2021) 397:94-5. doi: 10.1016/S0140-6736(20)32515-0

33. Ellul MA, Benjamin L, Singh B, Lant S, Michael BD, Easton A, et al. Neurological associations of COVID-19. Lancet Neurol. (2020) 19:76783. doi: 10.1016/S1474-4422(20)30221-0

34. Becerra-Muñoz VM, Núñez-Gil IJ, Eid CM, Aguado MG, Romero R, Huang J, et al. Clinical profile and predictors of in-hospital mortality among older patients admitted for COVID-19. Age Ageing. (2020) 50:32634. doi: 10.1093/ageing/afaa258

35. Ren L, Yu S, Xu W, Overton JL, Chiamvimonvat N, Thai PN. Lack of association of antihypertensive drugs with the risk and severity of COVID-19: a meta-analysis. J Cardiol. (2020) 77:482-91. doi: 10.1016/j.jjcc.2020.10.015

36. Yu MH, Wu XX, Chen CL, Tang SJ, Jin JD, Zhong CL, et al. Disseminated Nocardia infection with a lesion occupying the intracranial space complicated with coma: a case report. BMC Infect Dis. (2020) 20:856. doi: 10.1186/s12879-020-05569-4

37. Ma C, Cong Y, Zhang H. COVID-19 and the digestive system. Am J Gastroenterol. (2020) 115:1003-6. doi: 10.14309/ajg.0000000000000691

38. Huang C, Wang Y, Li X, Ren L, Zhao J, Hu Y, et al. Clinical features of patients infected with 2019 novel coronavirus in Wuhan, China. Lancet. (2020) 395:497-506. doi: 10.1016/S0140-6736(20)30183-5

39. Ye Q, Wang B, Zhang T, Xu J, Shang S. The mechanism and treatment of gastrointestinal symptoms in patients with COVID-19. Am J Physiol Gastrointest Liver Physiol. (2020) 319:G245-52. doi: 10.1152/ajpgi.00148.2020 
40. Zuo T, Zhang F, Lui GCY, Yeoh YK, Li AYL, Zhan H, et al. Alterations in gut microbiota of patients with COVID-19 during time of hospitalization. Gastroenterology. (2020) 159:944-55.e8. doi: 10.1053/j.gastro.2020.05.048

41. Wu Y, Cheng X, Jiang G, Tang H, Ming S, Tang L, et al. Altered oral and gut microbiota and its association with SARS-CoV-2 viral load in COVID-19 patients during hospitalization. NPJ Biofilms Microbiomes. (2021) 7:61. doi: 10.1038/s41522-021-00232-5

42. d'Ettorre G, Ceccarelli G, Marazzato M, Campagna G, Pinacchio C, Alessandri F, et al. Challenges in the management of SARS-CoV2 infection: the role of oral bacteriotherapy as complementary therapeutic strategy to avoid the progression of COVID-19. Front Med. (2020) 7:389. doi: $10.3389 /$ fmed.2020.00389

43. Walton GE, Gibson GR, Hunter KA. Mechanisms linking the human gut microbiome to prophylactic and treatment strategies for COVID-19. $\mathrm{Br} \mathrm{J}$ Nutr. (2021) 126:219-27. doi: 10.1017/S0007114520003980

44. Stavropoulou E, Bezirtzoglou E. Probiotics in medicine: a long debate. Front Immunol. (2020) 11:2192. doi: 10.3389/fimmu.2020.02192

45. Mak JWY, Chan FKL, Ng SC. Probiotics and COVID-19: one size does not fit all. Lancet Gastroenterol Hepatol. (2020) 5:644-5. doi: 10.1016/S2468-1253(20)30122-9

46. Hashimoto T, Perlot T, Rehman A, Trichereau J, Ishiguro H, Paolino M, et al. ACE2 links amino acid malnutrition to microbial ecology and intestinal inflammation. Nature. (2012) 487:477-81. doi: 10.1038/nature11228

47. Hersberger L, Bargetzi L, Bargetzi A, Tribolet P, Fehr R, Baechli V, et al. Nutritional risk screening (NRS 2002) is a strong and modifiable predictor risk score for short-term and long-term clinical outcomes: secondary analysis of a prospective randomised trial. Clin Nutr. (2020) 39:27209. doi: 10.1016/j.clnu.2019.11.041

48. Aguila EJT, Cua IHY, Fontanilla JAC, Yabut VLM, Causing MFP. Gastrointestinal manifestations of COVID-19: impact on nutrition practices. Nutr Clin Pract. (2020) 35:800-5. doi: 10.1002/ncp.10554

49. Wu J, Song S, Cao HC, Li LJ. Liver diseases in COVID-19: etiology, treatment and prognosis. World J Gastroenterol. (2020) 26:2286-93. doi: 10.3748/wjg.v26.i19.2286

50. Cheng Y, Luo R, Wang K, Zhang M, Wang Z, Dong L, et al. Kidney disease is associated with in-hospital death of patients with COVID-19. Kidney Int. (2020) 97:829-38. doi: 10.1016/j.kint.2020.03.005

51. Nadim MK, Forni LG, Mehta RL, Connor MJJr, Liu KD, Ostermann M, et al. COVID-19-associated acute kidney injury: consensus report of the 25th Acute Disease Quality Initiative (ADQI) Workgroup. Nat Rev Nephrol. (2020) 16:747-64. doi: 10.1038/s41581-020-00356-5

52. Ronco C, Reis T, Husain-Syed F. Management of acute kidney injury in patients with COVID-19. Lancet Respir Med. (2020) 8:738-42. doi: 10.1016/S2213-2600(20)30229-0

53. Li L, Zhang W, Hu Y, Tong X, Zheng S, Yang J, et al. Effect of convalescent plasma therapy on time to clinical improvement in patients with severe and life-threatening COVID-19: a randomized clinical trial. JAMA. (2020) 324:460-70. doi: 10.1001/jama.2020.10044

54. Janiaud P, Axfors C, Schmitt AM, Gloy V, Ebrahimi F, Hepprich M, et al. Association of convalescent plasma treatment with clinical outcomes in patients with COVID-19: a systematic review and meta-analysis. JAMA. (2021) 325:1185-95. doi: 10.1001/jama.2021.2747

55. Lundholm MD, Poku C, Emanuele N, Emanuele MA, Lopez N. SARSCoV-2 (COVID-19) and the endocrine system. J Endocr Soc. (2020) 4:bvaa144. doi: 10.1210/jendso/bvaa144

56. Pal R, Bhansali A. COVID-19, diabetes mellitus and ACE2: the conundrum. Diabetes Res Clin Pract. (2020) 162:108132. doi: 10.1016/j.diabres.2020.108132

57. Shekhar S, Wurth R, Kamilaris CDC, Eisenhofer G, Hannah-Shmouni F. Endocrine conditions and COVID-19. Hormone Metab Res. (2020) 52:47184. doi: $10.1055 / \mathrm{a}-1172-1352$

58. Pal R, Banerjee M. COVID-19 and the endocrine system: exploring the unexplored. J Endocrinol Invest. (2020) 43:102731. doi: 10.1007/s40618-020-01276-8

59. Bellastella G, Maiorino MI, Esposito K. Endocrine complications of COVID19: what happens to the thyroid and adrenal glands? J Endocrinol Invest. (2020) 43:1169-70. doi: 10.1007/s40618-020-01311-8

60. Corona G, Baldi E, Isidori AM, Paoli D, Pallotti F, De Santis L, et al. SARS-CoV-2 infection, male fertility and sperm cryopreservation: a position statement of the Italian Society of Andrology and Sexual Medicine (SIAMS) (Società Italiana di Andrologia e Medicina della Sessualità). J Endocrinol Invest. (2020) 43:1153-7. doi: 10.1007/s40618-020-01290-w

61. Li H, Xiao X, Zhang J, Zafar MI, Wu C, Long Y, et al. Impaired spermatogenesis in COVID-19 patients. EClinicalMedicine. (2020) 28:100604. doi: $10.1016 /$ j.eclinm.2020.100604

62. Pan F, Xiao X, Guo J, Song Y, Li H, Patel DP, et al. No evidence of severe acute respiratory syndrome-coronavirus 2 in semen of males recovering from coronavirus disease 2019. Fertil Steril. (2020) 113:11359. doi: 10.1016/j.fertnstert.2020.04.024

63. Chen H, Guo J, Wang C, Luo F, Yu X, Zhang W, et al. Clinical characteristics and intrauterine vertical transmission potential of COVID-19 infection in nine pregnant women: a retrospective review of medical records. Lancet. (2020) 395:809-15. doi: 10.1016/S0140-6736(20)30360-3

64. Chen L, Li Q, Zheng D, Jiang H, Wei Y, Zou L, et al. Clinical characteristics of pregnant women with COVID-19 in Wuhan, China. N Engl J Med. (2020) 382:e100. doi: 10.1056/NEJMc2009226

65. Ding Y, He L, Zhang Q, Huang Z, Che X, Hou J, et al. Organ distribution of severe acute respiratory syndrome (SARS) associated coronavirus (SARS$\mathrm{CoV}$ ) in SARS patients: implications for pathogenesis and virus transmission pathways. J Pathol. (2004) 203:622-30. doi: 10.1002/path.1560

66. Sansone A, Mollaioli D, Ciocca G, Limoncin E, Colonnello E, Vena W, et al. Addressing male sexual and reproductive health in the wake of COVID-19 outbreak. J Endocrinol Invest. (2020) 44:223-31. doi: 10.1007/s40618-020-01350-1

67. Qin C, Zhou L, Hu Z, Zhang S, Yang S, Tao Y, et al. Dysregulation of immune response in patients with coronavirus 2019 (COVID-19) in Wuhan, China. Clin Infect Dis. (2020) 71:762-8. doi: 10.1093/cid/ciaa248

68. Kucuk A, Cumhur Cure M, Cure E. Can COVID-19 cause myalgia with a completely different mechanism? A hypothesis. Clin Rheumatol. (2020) 39:2103-4. doi: 10.1007/s10067-020-05178-1

69. Carda S, Invernizzi M, Bavikatte G, Bensmail D, Bianchi F, Deltombe T, et al. COVID-19 pandemic. What should Physical and Rehabilitation Medicine specialists do? A clinician's perspective. Eur J Phy Rehabil Med. (2020) 56:51524. doi: 10.23736/S1973-9087.20.06317-0

70. Kelleni MT. Early use of non-steroidal anti-inflammatory drugs in COVID-19 might reverse pathogenesis, prevent complications and improve clinical outcomes. Biomed Pharmacother. (2020) 133:110982. doi: 10.1016/j.biopha.2020.110982

71. Sulli A, Gotelli E, Casabella A, Paolino S, Pizzorni C, Alessandri E, et al. Vitamin D and lung outcomes in elderly COVID-19 patients. Nutrients. (2021) 13:717. doi: 10.3390/nu13030717

72. Hernández JL, Nan D, Fernandez-Ayala M, García-Unzueta M, HernándezHernández MA, López-Hoyos M, et al. Vitamin D status in hospitalized patients with SARS-CoV-2 infection. J Clin Endocrinol Metab. (2021) 106:e1343-53. doi: 10.1210/clinem/dgaa733

73. Sabico S, Enani MA, Sheshah E, Aljohani NJ, Aldisi DA, Alotaibi NH, et al. Effects of a 2-Week 5000 IU versus 1000 IU vitamin D3 supplementation on recovery of symptoms in patients with mild to moderate COVID-19: a randomized clinical trial. Nutrients. (2021) 13:2170. doi: 10.3390/nu13072170

74. Murai IH, Fernandes AL, Sales LP, Pinto AJ, Goessler KF, Duran CSC, et al. Effect of a single high dose of vitamin D3 on hospital length of stay in patients with moderate to severe COVID-19: a randomized clinical trial. JAMA. (2021) 325:1053-60. doi: 10.1001/jama.2020.26848

75. The Lancet Diabetes Endocrinology. Vitamin D and COVID19: why the controversy? Lancet Diabetes Endocrinol. (2021) 9:53. doi: 10.1016/S2213-8587(21)00003-6

76. Disser NP, De Micheli AJ, Schonk MM, Konnaris MA, Piacentini AN, Edon DL, et al. Musculoskeletal consequences of COVID-19. J Bone Joint Surg Am Vol. (2020) 102:1197-204. doi: 10.2106/JBJS.20.00847

77. Gu R, Xu S, Li Z, Gu Y, Sun Z. The safety and effectiveness of rehabilitation exercises on COVID-19 patients: a protocol for systematic review and metaanalysis. Medicine. (2020) 99:e21373. doi: 10.1097/MD.0000000000021373

78. Reina-Gutiérrez S, Torres-Costoso A, Martínez-Vizcaíno V, Núñez de ArenasArroyo S, Fernández-Rodríguez R, Pozuelo-Carrascosa DP. Effectiveness of pulmonary rehabilitation in interstitial lung disease, including coronavirus diseases: a systematic review and meta-analysis. Arch Phys Med Rehabil. (2021) 102:1989-97.e3. doi: 10.1016/j.apmr.2021.03.035 
79. Li Z, Xie Z, Li H, Wang J, Wen X, Wu S, et al. Guidelines on the treatment with integrated traditional Chinese medicine and Western medicine for severe coronavirus disease 2019. Pharmacol Res. (2021) 174:105955. doi: 10.1016/j.phrs.2021.105955

80. Chen N, Zhou M, Dong X, Qu J, Gong F, Han Y, et al. Epidemiological and clinical characteristics of 99 cases of 2019 novel coronavirus pneumonia in Wuhan, China: a descriptive study. Lancet. (2020) 395:507-13. doi: 10.1016/S0140-6736(20)30211-7

81. Sava M, Sommer G, Daikeler T, Woischnig AK, Martinez AE, Leuzinger $\mathrm{K}$, et al. Ninety-day outcome of patients with severe COVID-19 treated with tocilizumab - a single centre cohort study. Swiss Med Wkly. (2021) 151:w20550. doi: 10.4414/smw.2021.20550

82. Lund LC, Kristensen KB, Reilev M, Christensen S, Thomsen RW, Christiansen CF, et al. Adverse outcomes and mortality in users of non-steroidal anti-inflammatory drugs who tested positive for SARS-CoV-2: a Danish nationwide cohort study. PLoS Med. (2020) 17:e1003308. doi: 10.1371/journal.pmed.1003308

83. Catalán IP, Martí CR, Sota DP, Álvarez AC, Gimeno MJE, Juana SF, et al. Corticosteroids for COVID-19 symptoms and quality of life at 1 year from admission. J Med Virol. (2021) 94:205-10. doi: 10.1002/jmv.27296

84. Krammer F, SARS-CoV-2 vaccines in development. Nature. (2020) 586:51627. doi: 10.1038/s41586-020-2798-3

85. Callaway E, Scores of coronavirus vaccines are in competition - how will scientists choose the best? Nature. (2020). doi: 10.1038/d41586-020-01247-2. [Epub ahead of print].

86. Tu YF, Chien CS, Yarmishyn AA, Lin YY, Luo YH, Lin YT, et al. A review of SARS-CoV-2 and the ongoing clinical trials. Int J Mol Sci. (2020) 21:2657. doi: 10.3390/ijms21072657

87. Folegatti PM, Ewer KJ, Aley PK, Angus B, Becker S, BelijRammerstorfer S, et al. Safety and immunogenicity of the ChAdOx1 nCoV-19 vaccine against SARS-CoV-2: a preliminary report of a phase 1/2, single-blind, randomised controlled trial. Lancet. (2020) 396:467-78. doi: 10.1016/S0140-6736(20)31604-4

88. Logunov DY, Dolzhikova IV, Shcheblyakov DV, Tukhvatulin AI, Zubkova OV, Dzharullaeva AS, et al. Safety and efficacy of an rAd26 and rAd5 vectorbased heterologous prime-boost COVID-19 vaccine: an interim analysis of a randomised controlled phase 3 trial in Russia. Lancet. (2021) 397:67181. doi: 10.1016/S0140-6736(21)00234-8

89. Gao Q, Bao L. Development of an inactivated vaccine candidate for SARSCoV-2. Science. (2020) 369:77-81. doi: 10.1126/science.abc1932

90. Xia S, Zhang Y, Wang Y, Wang H, Yang Y, Gao GF, et al. Safety and immunogenicity of an inactivated SARS-CoV-2 vaccine, BBIBP-CorV: a randomised, double-blind, placebo-controlled, phase 1/2 trial. Lancet Infect Dis. (2021) 21:39-51. doi: 10.1016/S1473-3099(20)30831-8

91. Richmond $\mathrm{P}$, Hatchuel L, Dong $\mathrm{M}$, $\mathrm{Ma} \mathrm{B}$, $\mathrm{Hu}$ B, Smolenov I, et al. Safety and immunogenicity of S-Trimer (SCB-2019), a protein subunit vaccine candidate for COVID-19 in healthy adults: a phase 1, randomised, double-blind, placebo-controlled trial. Lancet. (2021) 397:682-94. doi: 10.1016/S0140-6736(21)0 0241-5

92. Yang L, Tian D, Liu W. [Strategies for vaccine development of COVID-19]. Sheng $W u$ Gong Cheng Xue Bao. (2020) 36:593604. doi: 10.13345/j.cjb.200094

93. Polack FP, Thomas SJ, Kitchin N, Absalon J, Gurtman A, Lockhart S, et al. Safety and efficacy of the BNT162b2 mRNA COVID-19 vaccine. N Engl J Med. (2020) 383:2603-15. doi: 10.1056/NEJMoa2034577

94. Sherman SM, Smith LE, Sim J, Amlôt R, Cutts M, Dasch H, et al. COVID-19 vaccination intention in the UK: results from the COVID19 vaccination acceptability study (CoVAccS), a nationally representative cross-sectional survey. Hum Vaccin Immunother. (2021) 17:1612-621. doi: 10.1080/21645515.2020.1846397

95. Li G, Fan Y. Coronavirus infections and immune responses. Med Virol. (2020) 92:424-32. doi: 10.1002/jmv.25685

96. Devarakonda CKV, Meredith E. Coronavirus receptors as immune modulators. J Immunol. (2020) 206:923-9. doi: 10.4049/jimmunol.2001062

Conflict of Interest: The authors declare that the research was conducted in the absence of any commercial or financial relationships that could be construed as a potential conflict of interest.

Publisher's Note: All claims expressed in this article are solely those of the authors and do not necessarily represent those of their affiliated organizations, or those of the publisher, the editors and the reviewers. Any product that may be evaluated in this article, or claim that may be made by its manufacturer, is not guaranteed or endorsed by the publisher.

Copyright (c) 2021 Wang, Zhu, Xue, Wen and Tao. This is an open-access article distributed under the terms of the Creative Commons Attribution License (CC BY). The use, distribution or reproduction in other forums is permitted, provided the original author(s) and the copyright owner(s) are credited and that the original publication in this journal is cited, in accordance with accepted academic practice. No use, distribution or reproduction is permitted which does not comply with these terms. 